\title{
MRNA-Derived Prostate Cancer Vaccine CV9103
}

National Cancer Institute

\section{Source}

National Cancer Institute. mRNA-Derived Prostate Cancer Vaccine CV9103. NCI

Thesaurus. Code C82654.

A prostate cancer vaccine containing mRNAs encoding prostate specific antigen (PSA), prostate specific membrane antigen (PSMA), prostate stem cell antigen (PSCA) and sixtransmembrane epithelial antigen of the prostate (STEAP), with potential antitumor activity. Upon administration, mRNA-derived prostate cancer vaccine CV9103 may stimulate the immune system to mount a cytotoxic $T$ lymphocyte response (CTL) against PSA-, PSMA-, PSCA- and ST EAP-expressing prostate tumor cells. The mRNA used in this vaccine is modified and formulated to have enhanced translational potency and adjuvant activities. PSA, PSMA, PSCA and STEAP may be upregulated in prostate cancer cells; their expression in prostate cancer has been correlated with disease progression. 